\title{
Genes that are Affected in High Hydrostatic Pressure Treatments in a Listeria Monocytogenes Scott A ctsR Deletion Mutant
}

\author{
Yanhong Liu ${ }^{* 1}$ Lihan Huang ${ }^{2}$, Rolf D, Joerger ${ }^{3}$, Nereus W. Gunther IV
}

${ }^{1}$ Molecular Characterization of Foodborne Pathogen Research Unit, University of Delaware, USA

${ }^{2}$ Residue Chemistry and Predictive Microbiology Research Unit, Eastern Regional Research Center, 600 East Mermaid Lane, Wyndmoor, PA 19038, USA

${ }^{3}$ Department of Animal and Food Science, University of Delaware, Newark, DE19716, USA

\begin{abstract}
Listeria monocytogenes is a food-borne pathogen of significant threat to public health. High Hydrostatic Pressure (HHP) treatment can be used to control L. monocytogenes in food. The CtsR (class three stress gene repressor) protein negatively regulates the expression of class III heat shock genes. In a previous study, a spontaneous ctsR L. monocytogenes deletion mutant 2-1 that was able to survive under HHP treatment was identified; however, there is only limited information about the mechanisms of survival and adaptation of this mutant in response to high pressure. Microarray technology was used to monitor the gene expression profiles of cts $R$ mutant 2-1 under pressure treatments (450 Mpa, $3 \mathrm{~min})$. Some of the gene expression changes determined by microarray assays were confirmed by real-time RT-PCR analyses. Compared to non-pressure-treated ctsR mutant 2-1, 14 genes were induced ( $>2$-fold increase) in the ctsR deletion mutant whereas 219 genes were inhibited $(<-2$-fold decrease) by pressure treatments. The induced genes included genes encoding proteins involved in synthesis of purines, pyrimidines, nucleosides, and nucleotides, transport and binding, transcription, cell membrane, DNA and energy metabolism, protein synthesis, and unknown functions. The inhibited genes included genes encoding proteins for transport and binding, cell envelope, transcription, amino acid biosynthesis, regulatory functions, cellular processes and central intermediary metabolism. The information concerning $L$. monocytogenes survival under HHP at the molecular level may contribute to improved HHP treatments for food processing.
\end{abstract}

Keywords: Listeria monocytogenes; Scott A; microarray and realtime PCR; High hydrostatic pressure (HHP); ctsR mutant

\section{Introduction}

L. monocytogenes is a Gram-positive bacterium that can cause listeriosis in animal and human populations. Listeriosis is a foodborne disease with a high mortality rate (approximately 20 to $30 \%$ of cases) and occurs mostly in susceptible individuals such as pregnant women, newborns, the elderly, and immune-compromised patients. Outbreaks of listeriosis have been associated with the consumption of contaminated food products including ready-to-eat (RTE) meats and dairy products $[1,2]$. Because L. monocytogenes is widely distributed in the environment and survive under very harsh conditions, it is very difficult to eliminate this pathogen from foods and/or food processing plants.

High Hydrostatic processing (HHP) is a process that can inactivate microorganisms without significant deterioration of food quality. Foods treated with HPP generally have better sensory and nutritional qualities than products processed in more traditional ways. HHP has been used as a non-thermal preservation technique for processing of meats and dairy products to control L. monocytogenes and extend product shelf-life. In the food industry, pressures within the range of 300 to $600 \mathrm{MPa}$ are used to inactivate vegetative cells of microorganisms, including pathogens such as L. monocytogenes. However, the efficiency of HHP depends on the pressure, time, and composition of the food [3]. For example, the inactivation of L. monocytogenes by HHP (600 $\mathrm{MPa}, 5 \mathrm{~min}$ ) ranged from 1.82 to 3.85 Log units, depending on the type of dry-cured ham [4].

The pressure tolerance of $L$. monocytogenes is also growth-stage dependent. Stationary-phase cells are often more resistant to pressure than the exponential-phase cells [5]. High pressure resulted in changes in viability, morphology, and physiology in bacteria such as $E$. coli and L. monocytogenes [6-9]. However, the molecular survival mechanisms of L. monocytogenes under high pressure remain unknown.
Microarrays have been used to study differential gene expression of $L$. monocytogenes and E. coli during HPP and some important genes have been identified [10-13].

The $c t s R$ gene encodes a transcriptional regulator that represses the class III heat shock genes. CtsR has been shown to be related to high pressure since several pressure-tolerant mutants contained mutations in this gene [14-20]. L. monocytogenes Scott A ctsR mutant 2-1 exhibiting a higher level of viability under HPP and was less virulent, non-motile, heat and acid resistant, and sensitive to nisin [15]. Compared to the wild-type L. monocytogenes, genes that were differentially expressed in $c t s R$ mutant 2-1 under high pressure treatment were identified [12]. However, why the ctsR mutant 2-1 survives better under HHP treatments is unknown.

In the present paper, we compared gene expression of the $c t s R$ mutant 2-1 under HHP treatment vs. normal conditions. Our purpose was to explore what other genes contribute to the barotolerance in the $c t s R$ mutant 2-1. Since $c t s R$ mutants are most frequently isolated under high pressure treatments $[18,20]$, they represent a critical challenge in the tailing effect of HHP. Understanding the survival mechanism of the

*Corresponding author: Yanhong Liu, U.S. Department of Agriculture, Agricultura Research Service, Molecular Characterization of Foodborne Pathogen Research Unit, Eastern Regional Research Center, 600 East Mermaid Lane, Wyndmoor, PA 19038 USA. Tel: 215-233-6587; Fax: 215-233-6581; E-mail: Yanhong.Liu@ars.usda.gov

Received January 06, 2012; Accepted March 27, 2012; Published April 01, 2012

Citation: Liu Y, Huang L, Rolf D, Joerger, Gunther NW IV (2012) Genes that are Affected in High Hydrostatic Pressure Treatments in a Listeria Monocytogenes Scott A ctsR Deletion Mutant. J Microb Biochem Technol S2:003. doi:10.4172/19485948.S2-003

Copyright: (c) 2012 Liu Y, et al. This is an open-access article distributed unde the terms of the Creative Commons Attribution License, which permits unrestricted use, distribution, and reproduction in any medium, provided the original author and source are credited 
ctsR mutant under HHP may assist in developing strategies to reduce the tailing effects of HHP treatments.

\section{Materials and Methods}

\section{Bacterial strains and HHP treatments}

The L. monocytogenes Scott A ctsR mutant 2-1 and L. monocytogenes strain ScottA (wild-type) obtained from Dr. Joerger at University of Delaware were treated with high pressure ( $450 \mathrm{Mpa}, 3 \mathrm{~min}$ ) as described previously [12]. After pressure treatments, the suspension was centrifuged and the pellets were resuspended in RNAlater and followed by RNA isolation according to Liu et al., [12]. The L. monocytogenes Scott A ctsR mutant 2-1 using as control samples was held at room temperature at atmospheric pressure for 3 min before centrifugation.

RNA isolation, microarray chip design, hybridization, and data analysis

Total RNA was isolated and quantified as described previously [12]. A whole genome microarray was constructed as described previously [12]. All samples (both wild-type and the ctsR mutant 2-1) were hybridized twice with one experiment (chip 1) using Alexa Fluor 555 to label the cDNA under normal conditions and Alexa Fluro 647 to label cDNA under pressure treatment and in the reciprocal experiment (chip2), Alexa Fluor 647 was used to label the cDNA under normal conditions and Alexa Fluro 555 to label the cDNA under pressure treatment. Microarray hybridization and washing was performed and the microarray slide was scanned, quantified as described previously [12]. A minimum threshold of a 2 -fold change in gene expression with a p-value of $<0.01$ was used as the cut-off value.

\section{cDNA synthesis, primer design and real-time PCR analysis}

Synthesis of cDNA was carried out using Invitrogen's SuperScript III First-Strand Synthesis SuperMix (Invitrogen, Carlsbad, CA) following the manufacturer's instructions. Primers selected based on the gene sequences of L. monocytogenes F2365 strain (GenBank accession\#AE017262) were designed using Primer3 (v.0.4.0) software. Primer sequences are listed in Table 1 and Table 2. The housekeeping gene $(s p o G)$ was used as the internal control gene for real-time PCR analysis (Primer sequences 5'TGACGGTGAATTCCGTGATA3'; 5'TCAGCAGAAACGGATTCAGA3') since this gene had the least variation among other housekeeping genes including $16 \mathrm{~S}$ rRNA and gyrB (data not shown). PCR was performed in a 96-well plate on an Applied Biosystems 7500 Real-Time (ABI, Carlsbad, CA) PCR System as described previously [21]. To determine relative gene expression, the value of the internal control gene was subtracted from the pressure treated samples. The $\Delta \mathrm{Ct}, \Delta \Delta \mathrm{Ct}$, and the $2^{-\mathrm{fx}}$ values were calculated as previously described [21].

\section{Microarray data accession number}

The microarray data have been deposited into the Gene Expression Omnibus (GEO) database under accession number GSE32172 (www. ncbi.nlm.nih.gov/geo).

\section{Results}

\section{Barotolerance of L. monocytogenes Scott A wild type and the ctsR mutant 2-1}

The response of wild type L. monocytogenes Scott A and its ctsR mutant 2-1 to high pressure treatment (450 Mpa, 3 minutes) was investigated. At the pressure of $450 \mathrm{Mpa}$, the wild type exhibited a reduction in viability by $8.6-\log _{10}$ units, while the $c t s R$ mutant $2-1$ exhibited 6.7- $\log _{10}$ reduction in viability. Our data are consistent with the previously findings [15].

\section{Induced genes in L. monocytogenes Scott A ctsR mutant 2-1 strain under HHP treatment}

A total of 14 genes were expressed at higher levels in the $c t s R$ mutant 2-1 under HPP treatment. The genes that were expressed at higher levels in the wildtype under pressure were also identified using microarray assays (Data not shown). There are 7 genes present in both wildtype and the $c t s R$ mutant 2-1. The 7 unmatched genes that were only present in the ctsR mutant 2-1 are highlighted in boldface (Table 3). The unmatched genes are proposed to be pressure-resistant genes due to $c t s R$ deletion. These genes are grouped into the following categories: genes encoding for proteins involved in transcription, regulatory functions, cell envelope, DNA and energy metabolism, and unknown functions

LMOf2365_1986 encoding for a transcriptional regulator in the Fur family was expressed at a moderate level in the ctsR mutant 2-1 under HPP treatment (Table 3). This gene has been shown to be involved in barotolerance in L monocytogenes LO28 strains [22]. Disruption of the fur gene resulted in reduction of virulence, increased resistance to hydrogen peroxide and sensitivity to low-iron conditions [23].

LMOf2365_1515 encoding transcription elongation factor GreA was highly expressed in the ctsR mutant 2-1 under pressure. The elongation factor GreA binds to RNA polymerase and modulates transcriptional pausing. Deletion of this gene in E. coli resulted in

\begin{tabular}{|c|c|c|c|}
\hline GENE & Forward primer sequence & Reverse primer sequence & Amplicon size (bp) \\
\hline LMOf2365_0019 & TTCATCTTTGCGTTCATTCA & GATAAATGCGGCGAATAAAA & 111 \\
\hline LMOf2365_0992 & GCGCCTCGAGTTGTGTAATA & ATTTGATGAAGGCTTGCTTG & 146 \\
\hline LMOf2365_0993 & AATACATAATCGCGGAACCA & AGGTTACAGTGCCTTTGCAG & 150 \\
\hline LMOf2365_1036 & CTTAGTTCCCCCGTGGTTAT & CGCCAGAAATCTAAGTTCCA & 135 \\
\hline LMOf2365_1075 & TCGCAGCAGATACAGACCAC & CCGGCAGAACCGATATTAGA & 178 \\
\hline LMOf2365_1076 & CGCCCAAATACAGACGAAAT & AGCAGCGATTTTTGCTTCAT & 177 \\
\hline LMOf2365_1438 & CGCCGATAGAATAACCAATG & GATTTTAGGTTTCCCGCAAT & 122 \\
\hline LMOf2365_1515 & CGTCTTTTGCGGAATCATAC & ATGACCCTAGATGGGAAAGC & 145 \\
\hline LMOf2365_1844 & AATCACGTTCCGGTAACAAA & TTAGGTTTGCCGTTAACCAG & 103 \\
\hline LMOf2365_1920 & TCACGATCACCAAATGACAC & TACGTTCCCAAAACGGATAA & 134 \\
\hline LMOf2365_1986 & AAGAAAACTTCCTCGGCACT & GGACGCATTAAAGCACAACT & 119 \\
\hline LMOf2365_2230 & TAGACCGCGTTCATAATGGT & GCGTATCGAAGACCGACTAA & 109 \\
\hline LMOf2365_2305 & AACTCTGTGCTTCACGGTTC & ACAGGCAACGACAAAAGAAG & 119 \\
\hline LMOf2365_2584 & TCCGCCATCTAAATCACATT & ACGTCTTACAGGTCGTTGGA & 100 \\
\hline
\end{tabular}

Table 1: Oligonucleotides used for real-time PCR to evaluate induced genes. 


\begin{tabular}{|c|c|c|c|}
\hline GENE & Forward primer sequence & Reverse primer sequence & Amplicon size (bp) \\
\hline LMOf2365_0040 & AAGTGCTGTCGGCAAAGTAG & TAAATGACGACTGGGTGGTT & 101 \\
\hline LMOf2365_0255 & CTAGCGAGAAGCGGTGATAC & CTTTGAAAAGGCCAATCATC & 145 \\
\hline LMOf2365_0267 & GTATATCCGAAGGCCGATTT & CAGCTAGTTCCATCGCATCT & 118 \\
\hline LMOf2365_0305 & CGGCTGCAACTTCATCTAAT & GTGGAAGAAAAAGCAACGAA & 109 \\
\hline LMOf2365_0342 & TCССТCCAACTGATACGAAA & CTACAGATTCCGCCTCTTCA & 105 \\
\hline LMOf2365_0694 & AAAGGAACGGTCGGTATTTC & ATCGCCGAATGTTACTGTGT & 123 \\
\hline LMOf2365_0742 & TTGCAAGAAACAAACAGCAA & TGGATTTACGTTCGGAAAAG & 136 \\
\hline LMOf2365_1001 & GTTCATCGGCAGGAATAGTG & CGCTTGCTCCATCTACTGAT & 123 \\
\hline LMOf2365_1051 & CAAAAGGGCAAGTGTTTGTT & TCCCTGAACAAGTTTCCGTA & 122 \\
\hline LMOf2365_1056 & GGCTGTCATGTTCGGATTAC & TTTGCGCGAATACAGTACAA & 113 \\
\hline LMOf2365_1102 & TTTGGGATGTCGTTGTCAGA & TTCGCTTTAACGCTCGATTT & 180 \\
\hline LMOf2365_1445 & CGTCCGTCTGTTGAATAACC & GTGAAGGCGATGGCTATAAA & 142 \\
\hline LMOf2365_1705 & TTTTGCATCGCTACCTTTTC & CCGAAAGTGTTGGATAATCG & 129 \\
\hline LMOf2365_1744 & GGATCCAGTACTCGCTTCAA & AGCAGTTGCAGAAGCTGATT & 108 \\
\hline LMOf2365_2328 & ССССTAAGCCTAGAATTTGC & CAAAATGCCAAAAAGAGTCG & 122 \\
\hline LMOf2365_2333 & TACGCTGTGCACGATAAAAA & TATGTTTTTCGTCGGGGTTA & 127 \\
\hline LMOf2365_2407 & CGCTCGTCTCTCGTTAGTTC & ACGTTTGCTTCGAAAAGAGA & 150 \\
\hline LMOf2365_2550 & AAGTCACGCTACGGTTTCAC & GACCGCGTTGTCAATAGAGT & 102 \\
\hline LMOf2365_2610 & TCTACTGCAGCAACGTCTTG & GTTGACAAAGACGGCAAACT & 140 \\
\hline LMOf2365_2646 & CGCTGGGATTTTGTAAGTTG & AACGTGGACGCAGAAGTAAA & 114 \\
\hline LMOf2365_2647 & CGTTGGAAAGGTTTGTTCAC & AAAAGGAATGGTTTGGGTTC & 142 \\
\hline LMOf2365_2742 & TGGTGCTCGCCTAGATTAAG & AGCAATGGCGATTTACTCTG & 126 \\
\hline LMOf2365_2749 & ССАGСТССТСТААСТСАССА & GTTGATAGCGGGATTGTGAC & 130 \\
\hline LMOf2365_2763 & TTTCTGCATCAGGAAGCTCT & GTTTGCAAGAAAACGTGGTT & 138 \\
\hline LMOf2365_2285 & CACAAGTCCTCGTCATTCCT & TTGGCTAGACGGTTAAATGC & 115 \\
\hline LMOf2365_2805 & ATCTGCCAAACATCCTCAAA & CCAAGGAAACGCGATTATTA & 108 \\
\hline
\end{tabular}

Table 2: Oligonucleotides used for real-time PCR to evaluate repressed genes.

decreased replication-dependent recombination [24], indicating that this gene is required for recombination. Consistent with this, LMOf2365_1920 that encodes for recombination protein $U$ is also expressed highly in the ctsR mutant 2-1. Elevation of this gene suggests that HHP directly damages DNA in the ctsR mutant 2-1. Interestingly, a DNA recombination and repair gene $(r e c D)$ has been shown to be essential for high pressure growth in a deep-sea bacterium $[25,26]$.

Genes related to energy metabolism were induced in the $c t s R$ mutant 2-1 under HPP treatment. LMOf2365_1075 to 1076 encode for dihydrolipoamide acetyltransferase and dihydrolipoamide dehydragenase, respectively. Both genes are highly expressed in the $c t s R$ mutant 2-1. Other relatively highly induced genes included genes encoding for a putative membrane protein (LMOf2365_1438) and a hypothetical protein with unknown function (LMOf2365_2230). Why these genes are induced in the $c t s R$ mutant 2-1 under pressure remains unknown.

\section{Repressed genes in L. monocytogenes Scott A ctsR mutant 2-1 strain under HPP treatment}

Microarray analysis identified 219 genes that were repressed in the ctsR mutant 2-1 under high pressure treatment (Please see the supplemental table). The genes that were repressed in the wildtype under pressure treatment were also identified by microarray analysis (Data not shown). Of the 219 genes, 112 genes were repressed only in the ctsR mutant $2-1$, not in the wildtype (Please see the supplement table). These genes encode proteins involved in amino acid biosynthesis, cell membrane, synthesis of purines, pyrimidines, nucleosides, and nucleotides, DNA metabolism, regulatory functions, transcription, energy metabolism, biosynthesis of cofactors, prosthetic groups and carriers, protein synthesis, fatty acid and phospholipid metabolism, central intermediary metabolism, transport and binding, and hypothetical proteins with unknown function. Only genes that encode for proteins involved in amino acid biosynthesis, cell envelope, transcription, transport and binding, regulatory functions, central intermediary metabolism and cellular processes were confirmed using real-time PCR assays. The 18 genes that were only repressed in the ctsR mutant 2-1 under pressure are highlighted in boldface (Table 4). These unmatched genes are likely to be pressure-resistant related genes due to $c t s R$ deletion.

Expression of a gene related to flagella synthesis (LMOf2365 Imo0742) was reduced significantly in the ctsR mutant 2-1 under pressure (Table 4), this correlates with the absence of flagella and non-motile characteristics of $c t s R$ mutant 2-1 [12]. Consistent with our findings, flagella mRNA and protein were also reduced in the $c t s R$ mutant AK01 [17].

The expression of the RNA polymerase $\sigma-70$ factor gene (LMOf2365_0255) was inhibited in the ctsR mutant 2-1 (-10-fold in the microarray and -5 -fold by real-time PCR), possibly suggesting compensation for HPP-induced inhibition of RNA synthesis. Bacterial $\sigma-70$ factor directs RNA polymerase (RNAP) to specific promoter sites and starts transcription [27]. Interestingly, RNA polymerase $\sigma-70$ factor was required for stabilization of a deep-sea piezophillic bacterium under high-pressure conditions [28]. Furthermore, several transcription-associated genes were also inhibited, including those encoding proteins involved in transcription regulation, and termination/antitermination activities (Table 4).

Some genes related to the cell envelope were inhibited in the $c t s R$ mutant 2-1 under HPP treatment. For example, LMOf2365_0345 encoding for a leucine rich repeat domain/LPXTG-motif cell wall anchor domain protein was inhibited (-5-fold in microarray and $10-$ 
Citation: Liu Y, Huang L, Rolf D, Joerger, Gunther NW IV (2012) Genes that are Affected in High Hydrostatic Pressure Treatments in a Listeria Monocytogenes Scott A ctsR Deletion Mutant. J Microb Biochem Technol S2:003. doi:10.4172/1948-5948.S2-003

\begin{tabular}{|c|c|c|c|}
\hline Category/Gene & Function $^{b}$ & Fold change $^{c}$ Microarray $^{d}$ & RT-PCR \\
\hline \multicolumn{4}{|c|}{$\begin{array}{l}\text { Genes encoding proteins involved in transport and } \\
\text { binding }\end{array}$} \\
\hline $\begin{array}{l}\text { LMOf2365_2305 } \\
\text { LMOf2365_1036 }\end{array}$ & $\begin{array}{l}\text { PTS system; fructose-specific; IIABC component } \\
\text { glycine betaine/L-proline ABC transporter; permease protein }\end{array}$ & $\begin{array}{l}6.5 \\
2.1\end{array}$ & $\begin{array}{l}34.3 \\
7.7\end{array}$ \\
\hline \multicolumn{4}{|c|}{ Genes encoding proteins involved in cell envelope } \\
\hline $\begin{array}{l}\text { LMOf2365_0992 } \\
\text { LMOf2365_0993 } \\
\text { LMOf2365_1438 }\end{array}$ & $\begin{array}{l}\text { D-alanyl carrier protein } \\
\text { dltB protein } \\
\text { putative membrane protein }\end{array}$ & $\begin{array}{l}5.0 \\
3.2 \\
3.4\end{array}$ & $\begin{array}{l}4.6 \\
7.0 \\
6.3\end{array}$ \\
\hline \multicolumn{4}{|c|}{$\begin{array}{l}\text { Genes encoding proteins involved in DNA } \\
\text { metabolism }\end{array}$} \\
\hline LMOf2365_1920 & recombination protein $\mathrm{U}$ & 3.2 & 2.7 \\
\hline \multicolumn{4}{|c|}{$\begin{array}{l}\text { Genes encoding hypothetical or unknown function } \\
\text { proteins }\end{array}$} \\
\hline LMOf2365_2230 & hypothetical protein & 2.3 & 2.4 \\
\hline \multicolumn{4}{|c|}{ Genes encoding proteins involved in transcription } \\
\hline LMOf2365_1515 & transcription elongation factor GreA & 2.5 & 2.5 \\
\hline \multicolumn{4}{|c|}{$\begin{array}{l}\text { Genes encoding proteins involved in regulatory } \\
\text { functions }\end{array}$} \\
\hline LMOf2365_1986 & transcriptional regulator, Fur family & 2.0 & 2.8 \\
\hline \multicolumn{4}{|c|}{$\begin{array}{l}\text { Genes encoding proteins of purines, pyrimidines, } \\
\text { nucleosides, and nucleotides }\end{array}$} \\
\hline LMOf2365_2584 & adenylate kinase & 3.4 & 3.2 \\
\hline \multicolumn{4}{|c|}{$\begin{array}{l}\text { Genes encoding proteins involved in protein } \\
\text { synthesis }\end{array}$} \\
\hline LMOf2365_1844 & ribosomal protein L28 & 2.2 & 1.8 \\
\hline \multicolumn{4}{|c|}{$\begin{array}{l}\text { Genes encoding proteins involved in energy } \\
\text { metabolism }\end{array}$} \\
\hline $\begin{array}{l}\text { LMOf2365_1075 } \\
\text { LMOf2365_1076 } \\
\text { LMOf2365_0019 }\end{array}$ & $\begin{array}{l}\text { dihydrolipoamide acetyltransferase } \\
\text { dihydrolipoamide dehydrogenase } \\
\text { cytochrome aa3 quinol oxidase, subunit IV }\end{array}$ & $\begin{array}{l}2.6 \\
2.6 \\
1.2\end{array}$ & $\begin{array}{l}1.9 \\
4.1 \\
119\end{array}$ \\
\hline
\end{tabular}

aOnly the genes that met the stringent criteria for being up-regulated in the ctsR mutant 2-1 of $L$. monocytogenes

Scott A (i.e., fold change $>2$ fold; $p<0.01$ ) are listed here.

${ }^{b}$ Gene functions are based on annotations provided by TIGR (http://cmr.jcvi.org/tigr-scripts/CMR/CmrHomePage.cgi).

'Fold change indicates the transcript ratios of the ctsR mutant 2-1 between pressure treatment (450 Mpa, 3 minutes)

and normal conditions as determined by microarray and real-time PCR.

${ }^{d}$ Numbers are average values from two independent experiments.

eNumbers are average values from two independent experiments.

Table 3: Genes induced in L. monocytogenes strain ScottA ctsR mutant 2-1 under pressure treatment ( 450 Mpa, 3minutes) as identified by microarraya and real-time PCR analysis. Gene induced only in the ctsR mutant 2-1 (not in the wildtype) are in boldface.

fold by real-time PCR) in the ctsR mutant 2-1. Deletion of this gene in L. monocytogenes EDG strain resulted in greater susceptibility to nisin [29].

A number of genes encoding transport and binding proteins were inhibited in the ctsR mutant 2-1 under HHP treatment (Table 4). Interestingly, three of the five PTS systems that were inhibited in the ctsR mutant 2-1 are beta-glucoside-specific, indicating inhibition of the uptake of beta-glucosides under pressure. Gene expression of several $\mathrm{ABC}$ transporters was also inhibited under pressure. The substrates for these $\mathrm{ABC}$ transporters remain to be characterized.

Three genes encoding for amino acid biosynthesis were inhibited in the ctsR mutant 2-1 under pressure. Thus, reduction in amino acid synthesis may be related to the survival of the $c t s R$ mutant 2-1 under pressure.

\section{Discussion}

In this study, microarrays were used to identify genes that are differentially expressed in a pressure tolerant $c t s R$ mutant strain 2-1 under HPP treatment. The ctsR mutant 2-1 held under normal conditions (no high pressure treatment) was used as a control. The wildtype under normal and pressure-treated conditions were also investigated. After comparison, the genes that were induced or repressed only in the ctsR mutant 2-1 (highlighted in boldface in Supplement (Table, Table 3 and Table 4 ) were proposed to be pressureresistant related genes due to $c t s R$ deletion. All of the induced genes identified by microarray analysis in $c t s R$ mutant 2-1 were confirmed by quantitative reverse transcriptase real-time PCR (qRT-PCR). Some of the repressed genes identified by microarray analysis were confirmed by qRT-PCR. The gene expression changes in the ctsR mutant 2-1only may contribute to the barotolerance and adaptation/survival of the ctsR mutant 2-1 under pressure.

We chose our HPP treatment to mimic HPP exposure procedures and exposure times typically used for food processing. The conditions we used (450 Mpa, $3 \mathrm{~min}$ ) resulted in a $6.7 \log$ reduction of the $c t s R$ mutant 2-1 whereas a $8.6 \log$ reduction was observed in wild-type L. monocytogenes Scott A. The gene expression levels of the housekeeping gene $(s p o G)$ in the $c t s R$ mutant 2-1 remained the same under HPP vs. normal conditions, suggesting that RNA synthesis was not inhibited under these conditions. However, it has been shown that with increased pressure levels HHP combined with extended exposure times in L. monocytogenes [10], resulted in inhibition of RNA synthesis [30].

A problem observed during high pressure treatment is that a small portion of a bacterial population can be relatively resistant after a certain 
Citation: Liu Y, Huang L, Rolf D, Joerger, Gunther NW IV (2012) Genes that are Affected in High Hydrostatic Pressure Treatments in a Listeria Monocytogenes Scott A ctsR Deletion Mutant. J Microb Biochem Technol S2:003. doi:10.4172/1948-5948.S2-003

\begin{tabular}{|c|c|c|c|}
\hline Category/Gene & Function $^{\mathrm{b}}$ & Fold change $^{c}$ Microarray $^{d}$ & RT-PCR \\
\hline \multicolumn{4}{|l|}{ Amino acid biosynthesis } \\
\hline LMOf2365_0624 & O-acetylhomoserine (thiol)-lyase & -2.3 & -2.0 \\
\hline LMOf2365_1705 & 5-methyltetrahydropteroyltriglutamate--homocysteine S-methyltransferase & -4.3 & -3.3 \\
\hline LMOf2365_2285 & aspartate aminotransferase & -3.6 & -2.5 \\
\hline $\begin{array}{l}\text { Cell envelope } \\
\text { LMOf2365_0342 }\end{array}$ & putative lipoprotein & -4.0 & -1.4 \\
\hline LMOf2365_0345 & leucine rich repeat domain/ LPXTG-motif cell wall anchor domain protein & -6.3 & -10 \\
\hline \multirow{5}{*}{$\begin{array}{l}\text { LMOf2365_0694 } \\
\text { LMOf2365_1102 } \\
\text { LMOf2365_2550 } \\
\text { LMOf2365_2610 } \\
\text { LMOf2365_2742 }\end{array}$} & cell wall surface anchor family protein & -2.3 & -10 \\
\hline & glycosyl transferase, group 2 family protein & -3.6 & -1.4 \\
\hline & putative lipoprotein & -2.9 & -28.6 \\
\hline & putative lipoprotein & -3.0 & -5.0 \\
\hline & D-alanyl-D-alanine carboxypeptidase & -2.9 & -87.8 \\
\hline Transport and binding proteins & \multirow{2}{*}{ sugar $A B C$ transporter, sugar-binding protein } & \multirow{2}{*}{-5.6} & \multirow{2}{*}{-2.0} \\
\hline LMOf2365_0267 & & & \\
\hline LMOf2365_0305 & D-methionine $A B C$ transporter, D-methionine-binding protein & -3.6 & -2.0 \\
\hline LMOf2365_0390 & PTS system, beta-glucoside-specific, IIB component & -2.8 & -1.4 \\
\hline LMOf2365_1001 & ABC transporter, permease protein & -5.0 & -3.3 \\
\hline LMOf2365_1056 & PTS system; beta-glucoside-specific; IIABC component & -7.7 & -2.5 \\
\hline LMOf2365_1445 & $\begin{array}{l}\text { glycine betaine/L-proline ABC transporter, glycine betaine/L-proline-binding } \\
\text { protein }\end{array}$ & -5.6 & -10 \\
\hline LMOf2365_1744 & PTS system, beta-glucoside-specific, IIB component & -6.3 & -2.5 \\
\hline LMOf2365_2247 & $A B C$ transporter, permease protein & -2.0 & -1.7 \\
\hline LMOf2365_2260 & ABC transporter, ATP-binding protein & -5.0 & -2.5 \\
\hline LMOf2365_2333 & amino acid antiporter & -3.6 & -2.0 \\
\hline LMOf2365_2646 & putative PTS system, galactitol-specific, IIB component & -3.4 & -2.0 \\
\hline LMOf2365_2647 & PTS system, IIA component & -4.0 & -5.0 \\
\hline LMOf2365_2749 & ABC transporter, ATP-binding protein & -2.3 & -1.4 \\
\hline \multicolumn{2}{|l|}{ Transcription } & \multirow{2}{*}{-7.1} & \multirow{2}{*}{-5.0} \\
\hline LMOf2365_0255 & RNA polymerase sigma-70 factor & & \\
\hline \multicolumn{4}{|l|}{ Regulatory functions } \\
\hline LMOf2365_0040 & transcriptional regulator; Lacl family & -3.3 & -1.3 \\
\hline LMOf2365_0344 & putative transcriptional activator & -2.3 & -1.7 \\
\hline LMOf2365_1051 & transcriptional regulator, Lacl family & -2.3 & -1.4 \\
\hline LMOf2365_2407 & transcription antiterminator LicT & -2.1 & -5.0 \\
\hline LMOf2365_2763 & transcription antiterminator, BglG family & -3.4 & -2.5 \\
\hline LMOf2365_2805 & transcriptional regulator, TetR family & -2.9 & -1.4 \\
\hline \multicolumn{4}{|l|}{ Central intermediary metabolism } \\
\hline LMOf2365_2328 & putative glucosamine-6-phosphate isomerase & -2.3 & -2.0 \\
\hline \multicolumn{4}{|l|}{ Cellular processes } \\
\hline LMOf2365_0742 & putative flagellar hook-associated protein FlgL & -2.1 & -2.5 \\
\hline
\end{tabular}

aOnly the genes that met the stringent criteria for being up-regulated in the ctsR mutant 2-1 of L. monocytogenes

Scott $A$ (i.e., fold change $>2$ fold; $p<0.01$ ) are listed here.

${ }^{b}$ Gene functions are based on annotations provided by TIGR (http://cmr.jcvi.org/tigr-scripts/CMR/CmrHomePage.cgi).

'Fold change indicates the transcript ratios of the ctsR mutant 2-1 between pressure treatment (450 Mpa, 3 minutes)

and normal conditions as determined by microarray and real-time PCR.

${ }^{\mathrm{d} N u m b e r s}$ are average values from two independent experiments.

eNumbers are average values from two independent experiments.

Table 4: Genes repressed in L. monocytogenes strain ScottA ctsR mutant 2-1 under pressure treatment as identified by microarray and real-time PCR analysis. Gene repressed only in the ctsR mutant 2-1 (not in the wildtype) are in boldface.

applied pressure. This phenomenon is called the tailing effect [31], and it is a major challenge for the food industry. There are indications that high pressure results in genetic changes in the pressure-resistant subpopulation. A majority of pressure-resistant mutants contained mutations in the $c t s R$ gene [16,18-20], indicating the involvement of this gene in the tailing effect. Understanding how the ctsR mutant 2-1 survives under HHP may help develop better strategies to eliminate the tailing effect of HHP in food processing. For example, LMOf2365_0345 encoding for a leucine rich repeat domain/LPXTG-motif cell wall anchor domain protein was repressed in the $c t s R$ mutant 2-1. Since deletion of this gene resulted in greater sensitivity to nisin [29], the reduced expression of LMOf2365_0345 in ctsR mutant 2-1 provides an explanation for the sensitivity of this mutant to nisin under high pressure. This suggests that combination of a nisin and HPP treatment may inhibit the growth of L. monocytogenes. This notion has been supported by a study showing that a combination of high pressure treatment with nisin inhibited the growth of L. monocytogenes [4], in dry-cured ham, therefore, preventing the tailing effect.

Although the enhanced barotolerance of the $c t s R$ mutant 2-1 made 
it difficult to eliminate the HHP tailing effect, the enhanced stress tolerance feature of the $c t s R$ mutant can be beneficial to lactic acid bacteria. Various $c t s R$ deletion mutants have been used successfully in food and beverage fermentation. For example, the $c t s R$ deletion mutant of Lactobacillus plantarum was shown to survive better under ethanol stress [32], suggesting that this mutant can be potentially used for making wine. In another study, a ctsR deletion mutant of Lactobacillis sakei improved raw sausage fermentation since it grew better under stress $[33,34]$.

Several lines of evidence suggest that the $c t s R$ mutant 2-1 and deepsea bacteria are similar in terms of pressure tolerance. First, the stress related genes were expressed under normal conditions. i.e. no pressure treatment. In the $c t s R$ mutant 2-1, the $c l p C$ operon was highly expressed; whereas in a deep-sea bacterium, stress related genes were also highly expressed [35]. Although the expressed stress genes were different in the $c t s R$ mutant 2-1 and a deep-sea bacterium, they may represent the same mechanism to compromise the environment. Second, some genes that were induced in the ctsR mutant 2-1 under pressure were also found to be necessary in deep-sea bacteria under pressure. e.g. genes encoding for respiratory chain [35] and recombinant proteins [25]. This indicates that they may share some adaptation/survival strategies under high pressure.

In the present study, whole-genome microarrays were used to identify multiple genes that were induced or inhibited by HHP treatment in $c t s R$ mutant 2-1. The induced genes and a portion of the repressed genes were confirmed by real-time PCR. Identification of these genes begins to reveal the molecular mechanisms responsible for the adaptation and survival of $c t s R$ mutant 2-1 under HHP treatment. Our results will provide a useful list of genes as novel candidates for probing the molecular mechanism and physiology of the stress response. On the basis of proven or putative function, we provide an interpretation and speculation on these detected changes in gene transcription. Further studies including the creation of gene knockouts need to be performed to confirm/identify the actual function of these genes.

\section{Acknowledgments}

We thank Amy Ream for performing real-time PCR assays. We are grateful to Anna Porto-Fett, John Luchansky, Brad Shoyer, and Jeffery Call for their work on HHP treatments. We appreciate Dr. Pina Fratamico and Dr. James Smith (USDA Agricultural Research Service, Eastern Regional Research Center, Wyndmoor $\mathrm{PA})$ for critical reading of the manuscript.

\section{References}

1. Farber JM, Peterkin PI (1991) Listeria monocytogenes, a food-borne pathogen Microbiol Rev 55: 476-511.

2. Fleming DW, Cochi SL, MacDonald KL, Brondum J, Hayes PS, et al. (1985) Pasteurized milk as a vehicle of infection in an outbreak of listeriosis. N Engl J Med 312: 404-407.

3. Patterson MF (2005) Microbiology of pressure-treated foods. J Appl Microbiol 98: 1400-1409.

4. Hereu A, Bover-Cid S, Garriga M, Aymerich T (2012) High hydrostatic pressure and biopreservation of dry-cured ham to meet the Food Safety Objectives for Listeria monocytogenes. Int J Food Microbiol 154: 107-112

5. McClements JM, Patterson MF, Linton M (2001) The effect of growth stage and growth temperature on high hydrostatic pressure inactivation of some psychrotrophic bacteria in milk. J Food Prot 64: 514-522.

6. Mañas P, Mackey BM (2004) Morphological and physiological changes induced by high hydrostatic pressure in exponential- and stationary-phase cells of Escherichia coli: relationship with cell death. Appl Environ Microbio 70: $1545-1554$

7. Niven GW, Miles CA, Mackey BM (1999) The effects of hydrostatic pressure on ribosome conformation in Escherichia coli: and in vivo study using differential scanning calorimetry. Microbiology 145: 419-425

8. Ritz M, Tholozan JL, Federighi M, Pilet MF (2001) Morphological and physiological characterization of Listeria monocytogenes subjected to high hydrostatic pressure. Appl Environ Microbiol 67: 2240-2247.

9. Ritz M, Tholozan JL, Federighi M, Pilet MF (2002) Physiological damages of Listeria monocytogenes treated by high hydrostatic pressure. Int $\mathrm{J}$ Food Microbiol 79: 47-53.

10. Bowman JP, Bittencourt CR, Ross $T$ (2008) Differential gene expression of Listeria monocytogenes during high hydrostatic pressure processing Microbiology 154: 462-475

11. Ishii A, Oshima T, Sato T, Nakasone K, Mori H, et al. (2005) Analysis of hydrostatic pressure effects on transcription in Escherichia coli by DNA microarray procedure. Extremophiles 9: 65-73

12. Liu Y, Ream A, Liu J, Wang Y (2011) Gene expression profiling of a pressure tolerant Listeria monocytogenes Scott A ctsR deletion mutant. J Ind Microbio Biotechnol 38: 1523-1533.

13. Malone AS, Chung YK, Yousef AE (2006) Genes of Escherichia coli O157:H7 that are involved in high-pressure resistance. Appl Environ Microbiol 72: 2661 2671.

14. Hu Y, Raengpradub S, Schwab U, Loss C, Orsi RH, et al. (2007) Phenotypic and transcriptomic analyses demonstrate interactions between the transcriptional regulators CtsR and Sigma B in Listeria monocytogenes. Appl Environ Microbiol 73: 7967-7980.

15. Joerger RD, Chen $\mathrm{H}$, Kniel KE (2006) Characterization of a spontaneous, pressure-tolerant Listeria monocytogenes Scott A ctsR deletion mutant. Foodborne Pathog Dis 3: 196-202.

16. Karatzas KA, Bennik MH (2002) Characterization of a Listeria monocytogenes Scott $A$ isolate with high tolerance towards high hydrostatic pressure. App Environ Microbiol 68: 3183-3189.

17. Karatzas KA, Wouters JA, Gahan CG, Hill C, Abee T, et al. (2003) The CtsR regulator of Listeria monocytogenes contains a variant glycine repeat region that affects piezotolerance, stress resistance, motility and virulence. Mol Microbiol 49: 1227-1238.

18. Karatzas KA, Valdramidis VP, Wells-Bennik MH (2005) Contingency locus in ctsR of Listeria monocytogenes Scott A: a strategy for occurrence of abundant piezotolerant isolates within clonal populations. Appl Environ Microbiol 71 8390-8396.

19. Van Boeijen IK, Chavaroche AA, Valderrama WB, Moezelaar R, Zwietering $M H$ et al. (2010) Population diversity of Listeria monocytogenes LO28: phenotypic and genotypic characterization of variants resistant to high hydrostatic pressure. Appl Environ Microbiol 76: 2225-2233.

20. Van Boeijen IK, Francke C, Moezelaar R, Abee T, Zwietering MH (2011) Isolation of highly heat-resistant Listeria monocytogenes variants by use of a kinetic modeling-based sampling scheme. Appl Environ Microbiol 77: 26172624.

21. Liu Y, Ream A (2008) Gene expression profiling of Listeria monocytogenes strain F2365 during growth in ultrahigh-temperature-processed skim milk. App Environ Microbiol 74: 6859-6866.

22. Considine KM, Sleator RD, Kelly AL, Fitzgerald GF, Hill C (2011) Novel listeria genetic loci conferring enhanced barotolerance in Escherichia coli. J App Microbiol 110: 618-630.

23. Rea RB, Gahan CG, Hill C (2004) Disruption of putative regulatory loci in Listeria monocytogenes demonstrates a significant role for Fur and PerR in virulence. Infect Immun 72: 717-727.

24. Poteete AR (2011) Recombination phenotypes of Escherichia coli greA mutants. BMC Mol Biol 12: 12.

25. Bidle KA Bartlett DH (1999) RecD function is required for high-pressure growth of a deep-sea bacterium. J Bacteriol 181: 2330-2337.

26. Bartlett DH (1999) Microbial adaptations to the psychrosphere/piezosphere. J Mol Microbiol Biotechnol 1: 93-100

27. Ghosh T, Bose D, Zhang X (2010) Mechanisms for activating bacterial RNA polymerase. FEMS Microbiol Rev 34: 611-627.

28. Kawano H, Nakasone K, Matsumoto M, Yoshida Y, Usami R, et al. (2004) 
Citation: Liu Y, Huang L, Rolf D, Joerger, Gunther NW IV (2012) Genes that are Affected in High Hydrostatic Pressure Treatments in a Listeria Monocytogenes Scott A ctsR Deletion Mutant. J Microb Biochem Technol S2:003. doi:10.4172/1948-5948.S2-003

Differential pressure resistance in the activity of RNA polymerase isolated from Shewanella violacea and Escherichia coli. Extremophiles 8: 367-375.

29. Popowska M, Markiewicz Z (2006) Characterization of Listeria monocytogenes protein Lmo0327 with murein hydrolase activity. Arch Microbiol 186: 69-86.

30. Yayanos AA, Pollard EC (1969) A study of the effects of hydrostatic pressure on macromolecular synthesis in Escherichia coli. Biophys J 9: 1464-1482.

31. Metrick C, Hoover DG, Farkas DF (1989) Effects of high hydrostatic pressure on heat-resistant and heat-sensitive strains of Salmonella. J Food Sci 54: 15471549.

32. Van Bokhorst-van de Veen $\mathrm{H}$, Abee T, Tempelaars M, Bron PA, Kleerebezem $\mathrm{M}$, (2011) Short- and long-term adaptation to ethanol stress and its cross- protective consequences in Lactobacillus plantarum. Appl Environ Microbio 77: 5247-5256.

33. Hüfner E, Hertel C (2008) Improvement of raw sausage fermentation by stressconditioning of the starter organism Lactobacillus sakei. Curr Microbiol 57: 490496.

34. Hüfner E, Markieton T, Chaillou S, Crutz-Le Coq AM, Zagorec M, et al. (2007) Identification of Lactobacillus sakei genes induced during meat fermentation and their role in survival and growth. Appl Environ Microbiol 73: 2522-2531.

35. Vezzi A, Campanaro S, D'Angelo M, Simonato F, Vitulo N, et al. (2005) Life at depth: Photobacterium profundum genome sequence and expression analysis. Science 307: 1459-1461.
This article was originally published in a special issue, Molecular diagnosis and detection technology handled by Editor(s). Prof. Jeffrey L. Ram, Wayne State University, USA; Dr. Lalitha Peddireddi, Kansas State University, USA; Dr. Liu Y, U.S. Department of Agriculture, Korea 